\title{
Developing a measure of dietary quality for the UK Biobank study
}

\author{
C. Montague ${ }^{1,2}$, S. D’angelo ${ }^{1}$, N.C. Harvey ${ }^{1,3}$, C. Vogel $^{1,3}$ and J. Baird ${ }^{1,3}$ \\ ${ }^{1}$ MRC Lifecourse Epidemiology Unit, Southampton General Hospital, University of Southampton, Southampton, UK, \\ ${ }^{2}$ Health Education England Wessex, Winchester, UK and \\ ${ }^{3}$ NIHR Southampton Biomedical Research Centre, University of Southampton and University Hospital Southampton \\ NHS Foundation Trust, Southampton, UK
}

The UK Biobank (UKB) has assessed diet among participants using a food frequency questionnaire (FFQ). Studies of UKB have tended to examine the association of intake of single foods with health outcomes. We used the FFQ data to develop a diet quality score that could be used to examine the relationship of dietary patterns with markers of cardiometabolic health.

We applied principal components analysis to the FFQ data for participants from the Foodscapes section of UKB $(n=52,345)$. The first component was transformed using Fisher-Yates normal scores, mapping it onto a Normal distribution with a mean of 0 and a standard deviation of 1 . The median value was used to categorise diet as healthy or unhealthy. We examined the relationship of the diet scores with markers of cardiometabolic health (blood pressure, serum lipids, BMI) in linear regression analyses, adjusting for confounding factors identified in a Directed Acyclic Graph: sex, age, ethnicity, educational attainment, smoking, Index of Multiple Deprivation.

Of the 52,345 potential participants, 33,149 (63\%) had complete dietary data. Just under half (45\%) were men. The average age was 56.2 years $(\mathrm{SD}=8.2)$. Most $(84 \%)$ were white and almost half had a college/university degree $(49.5 \%)$. The first component explained $14 \%$ of the variation in the diet data. It was characterised by high consumption of beef, any type of meat, white bread and low fibre cereals, and low intake of fresh/dried fruit, salad and cooked vegetables and was defined as an "unhealthy diet score". Coefficients were multiplied by -1 , so a positive value of the score indicated a healthy diet. A higher score, indicative of healthier diet, was associated with lower systolic and diastolic blood pressure $(\beta-0.81,95 \% \mathrm{CI}-1.0,-0.62 ; \beta-0.61,95 \% \mathrm{CI}-0.72,-0.05)$, lower BMI ( $\beta-0.67$, $95 \%$ CI $-0.73,-0.62$ ) and with healthier lipid profile (lower levels of cholesterol $\beta-0.05,95 \%$ CI $-0.06,-0.04$, triglycerides $\beta-0.05,95 \%$ CI $-0.06,-0.03$, and higher HDL cholesterol $\beta 0.01,95 \%$ CI $0.0,0.01)$. Participants with healthier diet scores $(>1)$ were less likely to be current smokers and more likely to be physically active $(>1$ /day vigorous activity) (both $\mathrm{P}<0.001)$.

The diet score was a good approximation of dietary quality and demonstrated a positive relationship between an unhealthy diet and markers of poorer cardiometabolic health. Our findings suggest that this measure of diet quality could be used to examine dietary patterns within the UKB and explore their relationship with a range of outcomes. 